\title{
Power-Performance Considerations of Parallel Computing on Chip Multiprocessors
}

\author{
JIAN LI and JOSÉ F. MARTÍNEZ \\ Cornell University
}

This paper looks at the power-performance implications of running parallel applications on chip multiprocessors (CMPs). First, we develop an analytical model that, for the first time, puts together parallel efficiency, granularity of parallelism, and voltage/frequency scaling, to establish a formal connection with the power consumption and performance of a parallel code running on a CMP. We then conduct detailed simulations of parallel applications running on a detailed powerperformance CMP model to confirm the analytical results and provide further insights. Both analytical and experimental models show that parallel computing can bring significant power savings and still meet a given performance target by choosing granularity and voltage/frequency levels judiciously. The particular choice, however, is dependent on the application's parallel efficiency curve and the process technology utilized, which our model captures. Likewise, analytical model and experiments show the effect of a limited power budget on the application's scalability curve. In particular, we show that a limited power budget can cause a rapid performance degradation beyond a number of cores, even in the case of applications with excellent scalability properties. On the other hand, our experiments show that, when a limited power budget is in place, power-thrifty memory-bound applications may actually enjoy better scalability than more computeintensive codes, even if the latter would exhibit higher scalability in a power-unconstrained scenario.

Categories and Subject Descriptors: C.1.4 [Processor Architectures]: Parallel Architectures

General Terms: Power, Performance, Parallel Computation, Chip Multiprocessors, Theory

Additional Key Words and Phrases: Voltage/frequency scaling, granularity, parallel efficiency

\section{INTRODUCTION}

Low-power computing has long been an important design objective for mobile, battery-operated devices. More recently, however, power consumption in high-performance microprocessors has drawn considerable attention from industry and researchers as well. Traditionally, power dissipation in CMOS technology has been significantly lower than other technologies, such as TTL or ECL. However, at current speeds and feature sizes, CMOS power consumption

Authors' address: Jian Li and José F. Martínez, Computer Systems Laboratory, Cornell University, Ithaca, NY 14853; email: \{li,martinez $\}$ csl.cornell.edu.

Permission to make digital or hard copies of part or all of this work for personal or classroom use is granted without fee provided that copies are not made or distributed for profit or direct commercial advantage and that copies show this notice on the first page or initial screen of a display along with the full citation. Copyrights for components of this work owned by others than ACM must be honored. Abstracting with credit is permitted. To copy otherwise, to republish, to post on servers, to redistribute to lists, or to use any component of this work in other works requires prior specific permission and/or a fee. Permissions may be requested from Publications Dept., ACM, Inc., 1515 Broadway, New York, NY 10036 USA, fax: +1 (212) 869-0481, or permissions@acm.org.

(C) 2005 ACM 1544-3566/05/1200-0397 $\$ 5.00$

ACM Transactions on Architecture and Code Optimization, Vol. 2, No. 4, December 2005, Pages 397-422. 
has increased dramatically. This makes microprocessor cooling increasingly difficult and expensive [Borkar 1999; Gunther et al. 2001]. As a result, over the last few years, power has become a first-priority concern to microprocessor designers/manufacturers [Agerwala and Chatterjee 2005; Weiser 2004].

In light of this mounting problem, industry and researchers are eyeing chip multiprocessor architectures (CMPs), which can attain higher performance by running multiple threads in parallel. By integrating multiple cores on a chip, designers hope to deliver performance growth while depending less on raw circuit speed and, thus, power [Agerwala and Chatterjee 2005].

Earlier VLSI works have discussed the trade-offs that sequential vs. parallel circuits present in silicon area and power consumption [Chandrakasan et al. 1992; Parhi 1999]. There is also rich literature on power/thermal-aware simultaneous multithreading (SMT) and CMP designs (or similar architecture configurations), most of which focuses on multiprogrammed workloads [Donald and Martonosi 2004; Ghiasi and Grunwald 2004; Kumar et al. 2003; Li et al. 2004; Sasanka et al. 2004; Seng et al. 2000]. So far, however, very little work has been done on the power-performance issues involving parallel applications executing on multiprocessors, in general and on multicore chips, in particular.

In a parallel run, processors synchronize and exchange data as they cooperate toward a common goal. Synchronization and communication constitute overheads that typically grow in importance as we increase the number of processors. This generally results in decreased parallel efficiency (speedup over number of processors used). (On the other hand, at low processor counts, the beneficial effect of the increased aggregate caching capacity over a single processor may yield a net increase in parallel efficiency-superlinear speedup.) As a result of the application's changing parallel efficiency, it is not obvious which voltage and frequency levels should be applied, in combination with the appropriate number of processors, to optimize a certain power-performance trade-off and/or meet a particular constraint. Overall, the general connective role that the application's parallel efficiency plays across processors in a parallel execution is not present in a multiprogrammed context, where processors operate largely independently of each other.

In this paper, we investigate the power-performance issues of running parallel applications on a CMP. First, we develop an analytical model to study the effect of the number of processors used, the parallel efficiency, and the voltage/frequency scaling applied, on the performance and power consumption delivered by a CMP. Specifically, we look at (1) optimizing power consumption given a performance target, (2) optimizing performance given a certain power budget, and (3) optimizing energy-delay product. Then, to confirm the insights developed from the analytical model and provide further insights, we conduct detailed simulations of parallel applications running on a power-performance model of a CMP. Finally, to shed some light in some of the specific opportunities and challenges that this study presents, we conduct a short simulation-based exploration of the powerperformance optimization space for one parallel application running on a CMP. 


\section{ANALYTICAL STUDY}

In this section, we develop an analytical model as a first approximation to studying the power-performance trade-offs specific to parallel computation on CMPs. First, we lay out the basic power and performance equations used in the model. We then study three important scenarios: (1) power optimization given a performance target; (2) performance optimization under a budget constraint; and (3) energy-delay product optimization.

\subsection{Power Equations}

Our power model is based on two well-known equations for CMOS logic [Kim et al. 2003; Mudge 2001]:

$$
\begin{gathered}
f_{\max }=\eta \frac{\left(V-V_{\mathrm{th}}\right)^{\alpha}}{V} \\
P=P_{\mathrm{D}}+P_{\mathrm{S}}=A C V^{2} f+V I_{\text {leak }}
\end{gathered}
$$

Equation (1) establishes the relationship between the supply voltage $V$ and the maximum operating frequency $f_{\max }$ where $V_{\text {th }}$ is the threshold voltage and $\eta$ and $\alpha$ are experimentally derived constants. Equation (2) defines power consumption $P$ as the sum of dynamic and static components, $P_{\mathrm{D}}$ and $P_{\mathrm{S}}$, respectively. In the dynamic term, $A$ is the gate activity factor, $C$ is the total capacitance, $V$ is the supply voltage, and $f$ is the operating frequency. In the static term, $V$ is again the supply voltage and $I_{\text {leak }}$ is the leakage current.

Leakage current mainly consists of subthreshold and gate-oxide leakage [Kim et al. 2003], $I_{\text {leak }}=I_{\text {sub }}+I_{\text {ox }}$. We comment on each one in turn.

$$
I_{\text {sub }}=K_{1} W e^{\frac{-V_{\text {th }} q}{n k(T+273)}}\left(1-e^{\frac{-V q}{k(T+273)}}\right)
$$

where $K_{1}$ and $n$ are experimentally derived constants, $W$ is the gate width, $V_{\text {th }}$ is threshold voltage, $k=1.38 \times 10^{-23} \mathrm{~J} / \mathrm{K}$ is Boltzmann's constant, $T$ is the temperature in degrees Celsius, $q=1.6 \times 10^{-19} \mathrm{C}$ is the electron unit charge, and $\mathrm{V}$ is the supply voltage.

$$
I_{\mathrm{ox}}=K_{2} W\left(\frac{V}{H_{\mathrm{ox}}}\right)^{2} e^{\frac{-\tau H_{\mathrm{ox}}}{V}}
$$

where $K_{2}$ and $\tau$ are experimentally derived constants, $H_{0 \mathrm{x}}$ is the oxide thickness, and $V$ is the supply voltage.

Thus, leakage current is largely exponentially dependent on $V$ and $T$. In our leakage current model, we approximate this dependency using a curve-fitted formula as follows:

$$
I_{\text {leak }}=I_{\text {leak,Vn,Tstd }} e^{a_{1}+a_{2} V+a_{3} T+a_{4} V T}=I_{\text {leak, }, \mathrm{nn}, \mathrm{Tstd}} e^{\phi(V, T)}
$$

where $I_{\text {leak,Vn,Tstd }}$ is the leakage current at nominal supply voltage $V_{\mathrm{n}}$ and room temperature $T_{\text {std }}\left(25^{\circ} \mathrm{C}\right)$, and $a_{1}, a_{2}, a_{3}$ and $a_{4}$ are curve-fitting constants. We use $\phi(V, T)$ to abbreviate the dependency of this curve-fitted formula on supply voltage and temperature. 
We validate our curve-fitted formula using HSpice simulations on a chain of inverters. In the HSpice simulations, we independently vary the supply voltage from a minimum of $2.3 V_{\text {th }}$ (to maintain enough noise margin [The ITRS Technology Working Groups 2003]) to $V_{\mathrm{n}}$, and the operating temperature from $25^{\circ}$ to $110^{\circ} \mathrm{C}$ for two process technologies: 130 and $65 \mathrm{~nm}$. The results show that the maximum error is within 9.5 and $7.5 \%$ for 130 and $65 \mathrm{~nm}$, respectively (0.25 and $0.05 \%$ average error, respectively).

Finally, we use the curve-fitted expression in Eq. (3) to replace $I_{\text {leak }}$ in Eq. (2) as follows:

$$
P=A C V^{2} f+V I_{\text {leak }, V_{\mathrm{n}}, T_{\mathrm{std}}} e^{\phi(V, T)}
$$

Our analytical model assumes a fixed CMP configuration built out of identical cores, each of which would comprise a microprocessor, some private cache(s), and a proportional part of the chip's interconnect. In this analytical model, in order to keep the discussion manageable, we assume a constant activity factor across the chip. This effectively excludes resources that have significantly lower switching activity and may occupy a large chip area, such as a L2 cache. In the experimental study, we make no such simplifications (Section 3).

\subsection{Performance Equations}

To model performance, we use the formula for execution time proposed in Hennessy and Patterson [2003]:

$$
t=I C C P I f^{-1}
$$

where $I C$ is the dynamic instruction count, $C P I$ is the average number of cycles per instruction, and $f$ is the operating frequency. Applications may run sequentially on one processor, or in parallel on $N$ processors (up to the maximum number of cores on the chip). When running in parallel on $N$ processors, we assume that all threads exhibit identical instruction counts and average number of clock cycles per instruction, $I C_{N}$ and $C P I_{N}$, respectively. Furthermore, we assume all processors use the same supply voltage $V_{N}$ and operating frequency $f_{N}$.

The parallel efficiency [Culler and Singh 1999] of an application running on $N$ processors $\varepsilon(N)$ can be written as:

$$
\varepsilon(N)=\frac{t_{1}}{N t_{N}}=\frac{I C_{1} C P I_{1} f_{1}^{-1}}{N I C_{N} C P I_{N} f_{N}^{-1}}
$$

If $f_{N}=f_{1}$ (i.e., no frequency scaling is applied), we can rewrite the parallel efficiency as:

$$
\varepsilon_{\mathrm{n}}(N)=\frac{I C_{1} C P I_{1}}{N I C_{N} C P I_{N}}
$$

We call this nominal parallel efficiency $\varepsilon_{\mathrm{n}}(N)$. It is a useful characterization of the application's parallel behavior on the CMP architecture, independent of 
power considerations. ${ }^{1}$ On the one hand, it captures the overheads of a parallel configuration, such as the communication overheads between threads, which can result in suboptimal performance gains $\left(\varepsilon_{\mathrm{n}}(N)<1\right)$. On the other hand, it also captures the beneficial effects of a parallel setup, such as increased aggregate caching capacity, which may result in superlinear performance gains $\left(\varepsilon_{\mathrm{n}}(N)>1\right)$.

Using these basic equations, we present models for three important scenarios: power optimization given a performance target (Section 2.3), performance optimization under a power budget constraint (Section 2.4), and energy-delay product optimization (Section 2.5).

\subsection{Scenario I: Power Optimization}

In this scenario, the goal is to find the configuration that maximizes power savings while delivering a prespecified level of performance. In particular, we require that all configurations deliver the performance of a sequential execution on one processor at full throttle, $t_{1}=t_{N}$. Thus, using Eq. 5, and for any number of processors $N$, we can write:

$$
I C_{1} C P I_{1} f_{1}^{-1}=I C_{N} C P I_{N} f_{N}^{-1}
$$

Using the definition of nominal parallel efficiency (Eq. 6), we can rewrite this equality as follows:

$$
f_{N}=\frac{f_{1}}{N \varepsilon_{\mathrm{n}}(N)}
$$

We can also rewrite Eq. (4) for the parallel case as follows:

$$
P_{N}=A N C V_{N}^{2} f_{N}+V_{N} N I_{\text {leak }, V_{\mathrm{n}}, T_{\text {std }}} e^{\phi\left(V_{N}, T_{N}\right)}
$$

where $N C$ and $N I_{\text {leak }, T_{\text {std }}}$ represent the aggregate capacitance and standard leakage current of the $N$-processor configuration, respectively. If we define the voltage scaling ratio $\vartheta=V_{N} / V_{1}$, we can rewrite Eq. (8) using $\vartheta$ and Eq. (7) as follows:

$$
\begin{aligned}
P_{N} & =A N C\left(\vartheta V_{1}\right)^{2} \frac{f_{1}}{N \varepsilon_{\mathrm{n}}(N)}+\left(\vartheta V_{1}\right) N I_{\text {leak }, T_{\mathrm{std}}} e^{\phi\left(\vartheta V_{1}, T_{N}\right)} \\
& =\frac{\vartheta^{2}}{\varepsilon_{\mathrm{n}}(N)} P_{\mathrm{D}, 1}+\vartheta N e^{\phi\left(\vartheta V_{1}, T_{N}\right)} P_{\mathrm{S}, 1, T_{\mathrm{std}}}
\end{aligned}
$$

where $P_{\mathrm{D}, 1}$ and $P_{\mathrm{S}, 1, T_{\text {std }}}$ are the dynamic and static power consumption at $N=1$, nominal voltage and frequency, and room temperature. We resort to published data from the International Technology Roadmap for Semiconductors (ITRS) [The ITRS Technology Working Groups 2003] to obtain $V_{1}, V_{\text {th }}, f_{1}$, $P_{\mathrm{D}, 1}$, and $P_{\mathrm{S}, 1, T_{\mathrm{std}}}$. We contemplate two process technologies, 130 and $65 \mathrm{~nm}$, and set the operating temperature of the single-core configuration at $T_{1}=100^{\circ} \mathrm{C}$. We assume voltage can scale continuously from the nominal supply voltage $V_{1}$ down to $2.3 V_{\text {th }}$. Frequency can scale continuously from $f_{1}$ without a lower

\footnotetext{
${ }^{1}$ We assume that $C P I_{N}$ does not depend on the clock frequency. In a system with multiple clocks (e.g., off-chip memory), this would require that any frequency scaling be applied to all clocks proportionally. Our experimental study does not make this simplification (Section 3).
} 
bound (always positive, of course). We set $A=1, \alpha=2$ [Mudge 2001]. We can obtain $\vartheta$ by solving the following equality derived from Eqs. (1) and (7):

$$
\frac{\left(\vartheta V_{1}-V_{\mathrm{th}}\right)^{2}}{\vartheta}=\frac{\left(V_{1}-V_{\mathrm{th}}\right)^{2}}{N \varepsilon_{\mathrm{n}}(N)}
$$

Using a 32-way CMP baseline, we study configurations running on different numbers of processor cores, assuming that unused processor cores in each case are shut down. In each configuration, we approximate the operating temperature $T_{N}$ using the HotSpot thermal model [Skadron et al. 2003] for its default Alpha EV6 floorplan, similarly to how we use it in our experimental study (Section 3).

We plot the normalized power consumption $P_{N} / P_{1}$ for each combination of process technology and operating temperature $T_{1}$, using $N=\{2,4,8,16,32\}$ processors, and nominal parallel efficiency ranging from $1 / N$ to 1 , in each case.

Notice that when $\varepsilon_{\mathrm{n}}(N)<\frac{1}{N}$, there is no way for the $N$-processor configuration to achieve the same performance as the single-processor one without raising $V_{N}$ over $V_{1}$. We do not allow this in the model. Also, although we do not plot regions with superlinear speedup $\left(\varepsilon_{\mathrm{n}}(N)>1\right)$, the results would be in line with the plots.

The curves (Figure 1) show that, for any $N$, higher nominal parallel efficiency $\varepsilon_{\mathrm{n}}(N)$ allows for greater power savings. Indeed, Eq. (7) shows that, as efficiency goes up, a lower frequency $f_{N}$ is required to maintain the performance of the single-core configuration. Generally, this also allows a lower supply voltage $V_{N}$ (Eq. 1). As a result, for a fixed $N$, higher efficiency results in lower dynamic and static power consumption (Eq. 8). Furthermore, a reduction in the dynamic activity results in lower die temperature $T_{N}$, which further reduces static power consumption (Eq. 8). This is captured in our analysis through the HotSpot thermal model [Skadron et al. 2003].

Recall, however, that to maintain acceptable noise margin, $V_{N}$ may not decrease below $2.3 V_{\text {th }}$. Thus, for a given $N$, there is an $\varepsilon_{\mathrm{n}}(N)$ beyond which the decrease in frequency is not accompanied by a decrease in supply voltage. This results in diminished returns on both dynamic and static power savings (Eq. 8). Moreover, the returns on static power savings are also limited by another lower bound: The die temperature can never be lower than the ambient temperature. In the plots, this is reflected by a change in the curvature.

In any case, for the configurations used, all curves show power savings with respect to the single-core configuration beyond a certain $\varepsilon_{\mathrm{n}}(N)$. Moreover, in general, the plots show that configurations with higher $N$ require a lower level of efficiency to reach their power break-even points. Equation (7) incorporates this trend: a higher $N$ makes $f_{N}$ (and thus power consumption) drop more rapidly as $\varepsilon_{\mathrm{n}}(N)$ increases.

Notice that, because $f_{N}$ drops more rapidly with larger $N$ (since we maintain the same performance target), voltage scaling can be more aggressive. This, however, also implies that the lower bound in supply voltage (and temperature) is reached (approached) earlier in the efficiency scale. As a result, scaling may not compensate for the extra power consumption introduced by the additional 

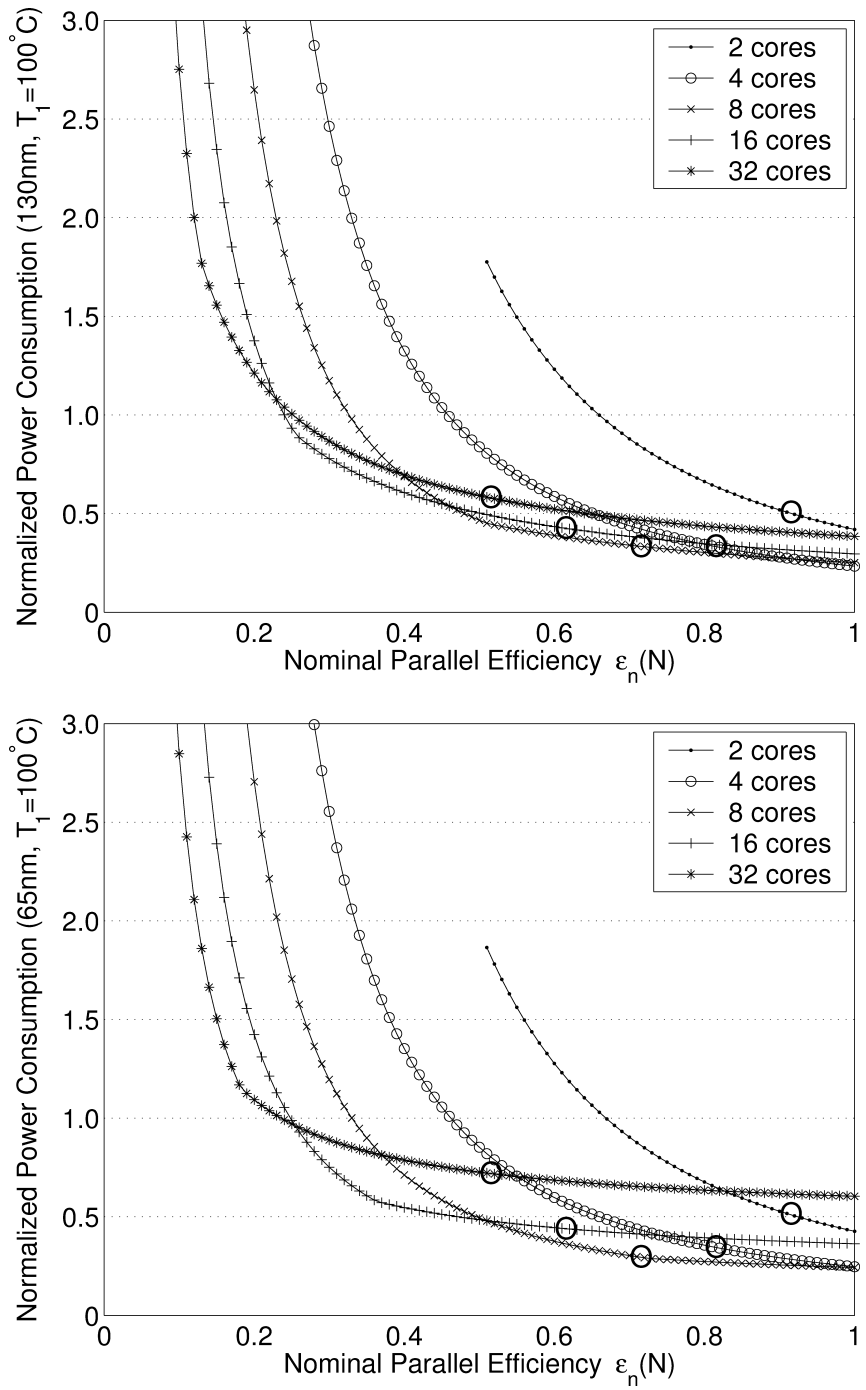

Fig. 1. Normalized power consumption using 130 (top) and $65 \mathrm{~nm}$ (bottom) technologies at $T_{1}=$ $100^{\circ} \mathrm{C}$, varying $N=\{2,4,8,16,32\}$ and $\varepsilon_{\mathrm{n}}(N) \in[1 / N, 1]$, and forcing that all configurations yield the performance of $N=1$ at full throttle. The marks in each plot indicate working points for an imaginary application with $\varepsilon_{\mathrm{n}}=\{0.9,0.8,0.7,0.6,0.5\}$.

cores. This is reflected in the plots by the fact that high- $N$ curves run above low- $N$ ones at high efficiency points. Overall, applications that exhibit a high degree of parallel efficiency do not necessarily save more power by choosing a larger $N$.

In any case, recall that $\varepsilon_{\mathrm{n}}(N)$ depends on $N$ and applications typically exhibit different levels of parallel efficiency with different $N$. (Usually, efficiency is lower with higher $N$ because of communication overhead.) Thus, for a given application, it generally does not make sense to directly compare the 
curves at a fixed point on the efficiency scale. To illustrate this, we mark in the plots the operating points of an imaginary application that exhibits $\varepsilon_{\mathrm{n}}=\{0.9,0.8,0.7,0.6,0.5\}$ for $N=\{2,4,8,16,32\}$, respectively. As we can see, the configuration that yields the maximum power savings is not necessarily the one with the highest number of processors, largely because of the decrease in parallel efficiency. This observation holds for both feature sizes.

Finally, it is important to note that, because absolute performance and power consumption is different in each of the two feature sizes, caution is advised when making comparisons across the plots.

\subsection{Scenario II: Performance Optimization}

In this scenario, the goal is to find the configuration that maximizes performance under a constrained power budget. In particular, we set the maximum power budget to that of executing on one processor at full throttle, $P_{1}=P_{N}$.

The performance gain or speedup $S$ on $N$ processors [Culler and Singh 1999] can be expressed as:

$$
S=\frac{t_{1}}{t_{N}}=\frac{I C_{1} C P I_{1} f_{1}^{-1}}{I C_{N} C P I_{N} f_{N}^{-1}}=N \varepsilon_{\mathrm{n}}(N) \frac{f_{1}^{-1}}{f_{N}^{-1}}
$$

Using Eq. (1) and the definition of voltage ratio $\vartheta$ (Section 2.3), we can rewrite the speedup as:

$$
S=N \varepsilon_{\mathrm{n}}(N) \frac{\left(\vartheta V_{1}-V_{\mathrm{th}}\right)^{\alpha}}{\vartheta\left(V_{1}-V_{\mathrm{th}}\right)^{\alpha}}
$$

To compute $\vartheta$, we introduce the problem restriction $P_{1}=P_{N}$. Using Eqs. (1), (4), and (8), we can express this restriction as:

$$
\begin{aligned}
& A C V_{1}^{2} \eta \frac{\left(V_{1}-V_{\text {th }}\right)^{\alpha}}{V_{1}}+V_{1} I_{\text {leak }, V_{\mathrm{n}}, T_{\text {std }}} e^{\phi\left(V_{1}, T_{1}\right)} \\
= & A N C\left(\vartheta V_{1}\right)^{2} \eta \frac{\left(\vartheta V_{1}-V_{\text {th }}\right)^{\alpha}}{\vartheta V_{1}}+\left(\vartheta V_{1}\right) N I_{\text {leak }, V_{\mathrm{n}}, T_{\text {std }}} e^{\phi\left(\vartheta V_{1}, T_{N}\right)}
\end{aligned}
$$

By using transformations similar to those used in Eq. (9), we obtain the equality:

$$
P_{\mathrm{D}, 1}+e^{\phi\left(V_{1}, T_{1}\right)} P_{\mathrm{S}, 1, T_{\mathrm{std}}}=N \vartheta \frac{\left(\vartheta V_{1}-V_{\mathrm{th}}\right)^{\alpha}}{\left(V_{1}-V_{\mathrm{th}}\right)^{\alpha}} P_{\mathrm{D}, 1}+\vartheta N e^{\phi\left(\vartheta V_{1}, T_{N}\right)} P_{\mathrm{S}, 1, T_{\mathrm{std}}}
$$

After obtaining $\vartheta$ from Eq. (11), we can resolve speedup $S$ in Eq. (10).

Figure 2 shows speedups for up to 32 cores under our constant power budget constraint. In the plot, we assume the application's nominal parallel efficiency $\varepsilon_{\mathrm{n}}(N)$ is 1 for any $N$. As before, we use two process technologies, $130 \mathrm{~nm}$ and $65 \mathrm{~nm}$.

It is interesting to observe that, even if the application's nominal parallel efficiency $\varepsilon_{\mathrm{n}}(N)$ is 1 , the maximum speedup achieved across all configurations is only a little over 4 , which corresponds to the configuration with $N=18$ and $130 \mathrm{~nm}$ process technology. Indeed, as the number of cores increases, the total 


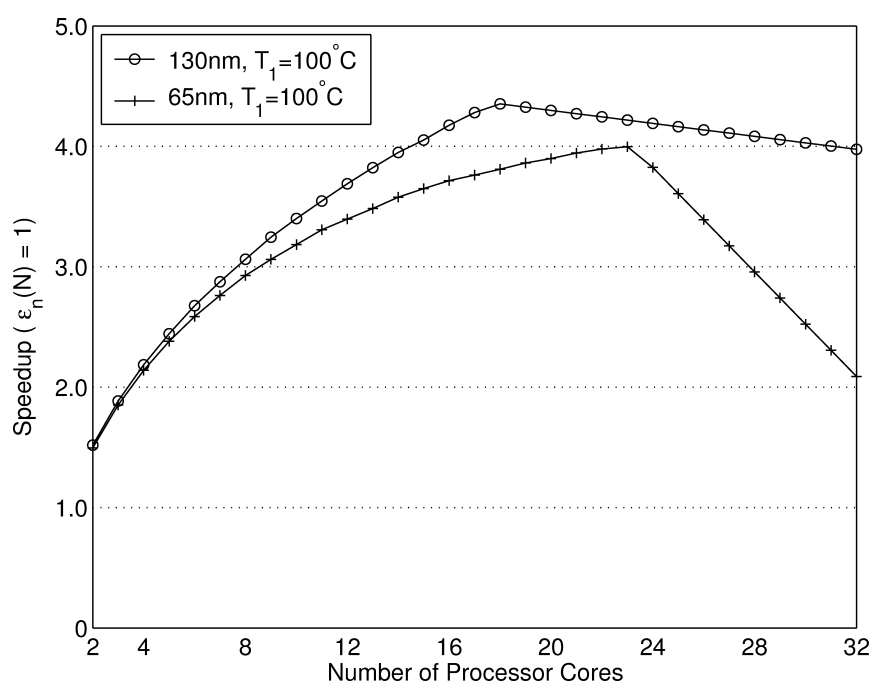

Fig. 2. Speedup of $N$-processor configurations with $\varepsilon_{\mathrm{n}}(N)=1$, and power budget in all cases equal to the power consumption of the single-processor configuration at full throttle.

power budget ought to remain constant. As a result, voltage/frequency scaling must be applied to the cores, which slows them down. The result is a suboptimal speedup with respect to the sequential execution.

Still, the speedup grows with the number of cores for relatively small configurations. For larger $N$, however, both technologies eventually show decreasing speedups. This is the case beyond $N=18$ and $N=23$ for 130 and $65 \mathrm{~nm}$, respectively. It means that, for even a perfectly scalable application, obtaining the optimum performance within a certain power budget may require a number of processors lower than the maximum number available. Indeed, due to the limited range of voltage scaling (Section 2.3 ), once the supply voltage reaches $2.3 V_{\text {th }}$ in our model, only frequency scaling can be applied further as the number of processors goes up. Unfortunately, dynamic power consumption is only linearly dependent on $f$ and thus the frequency reduction needed for each processor added is significant. This results in a significant performance degradation, most notably in the $65 \mathrm{~nm}$ case, where the ITRS data attributes a higher fraction of the total power consumption to static power [The ITRS Technology Working Groups 2003].

Note that, despite the speedup curve for $65 \mathrm{~nm}$ being below that of $130 \mathrm{~nm}$ at all times, the absolute performance of the $65 \mathrm{~nm}$ case might well be above that of $130 \mathrm{~nm}$ one. This is because each curve is relative to the performance of its respective sequential case. Also, note that, in this analysis, we tacitly assume that the application runs at maximum power in the sequential casein other words, the power consumption by the sequential setup is the power budget. In reality, however, applications may consume less than that when running sequentially, for example, if they stall often on memory accesses. In that case, as we assign more processors to the application, it would be possible to consume more than the power consumed by the sequential setup, and still be 
within budget. This may result in an extra performance "boost" beyond the gain predicted by the model. Thus, depending on the application characteristics, the speedups shown in the plots may be somewhat pessimistic. We consider this effect in the experimental study (Section 3).

\subsection{Scenario III: Energy-Delay Optimization}

In this scenario, the goal is to explore minimization of energy-delay product, $E t^{\beta}$, of a parallel run on a CMP. The energy-delay product captures both power and performance aspects of an execution. In general, by varying $\beta$, one can assign more weight to either $E$ or $t$, depending on the objective. On the one hand, $\beta>1$ puts more weight on performance. In particular, $\beta=2$ brings the problem closer to Scenario II's objective of optimizing performance, subject to a constant power constraint [Zyuban et al. 2004] (Section 2.4). On the other hand, $\beta<1$ emphasizes energy over performance. Specifically, $\beta=0$ degenerates into Scenario I (Section 2.3) - power optimization given a constant performance target (since $E=P t$, and $t$ is constant in this scenario). In this section, without loss of generality, we take the midpoint and choose $\beta=1$ [Gonzalez and Horowitz 1996].

We conduct a series of three analyses with increasing problem constraints. In the most optimistic case, we assume no limited power budget and a continuous voltage/frequency scaling function. We can write the energy-delay product of a parallel configuration of $N$ processors as:

$$
E_{N} t_{N}=P_{N} t_{N}^{2}=P_{N}\left(\frac{t_{1}}{S}\right)^{2} \propto \frac{P_{N}}{S^{2}}
$$

Using Eqs. (8) and (10), we can obtain the voltage and frequency levels that minimize the energy-delay product for a given $N$ and nominal parallel efficiency $\varepsilon_{\mathrm{n}}(N)$, by calculating the derivative of the above expression with respect to $V_{N}$. However, for the sake of simplicity (particularly in the later analysis, which impose additional constraints), we omit the (somewhat cumbersome) formulation here.

Instead, we scan the voltage/frequency range, and obtain the energy-delay product directly. In the first analysis, to approximate a continuous voltage/ frequency scaling function, we sample 1000 levels across the range. We select the minimum energy-delay product values, for both 130 and $65 \mathrm{~nm}$ process technologies, assuming $T_{1}=100^{\circ} \mathrm{C}, \varepsilon_{\mathrm{n}}(N)=1$, and $N \in[1,32]$. The top row of Figure 3 presents the results, normalized to the energy-delay product of the single-processor configuration at full throttle.

We plot curves for different values of parallel efficiency $\varepsilon_{\mathrm{n}}(N)$, constant across $N \in[2,32]$ (obviously, $\varepsilon_{\mathrm{n}}(1)=1$ ). In general, if parallel efficiency remains constant, adding more processors always results in a better energy-delay product, for both 130 and $65 \mathrm{~nm}$. In fact, the curve for the case $\varepsilon_{\mathrm{n}}(N)=1, N \in[1,32]$ largely follows a $1 / N$ shape.

Indeed, it can be shown that the per-core voltage and frequency levels are constant across this curve and equal to the levels that yield the optimum energydelay product for the single-processor case (which, as the plot shows, need not 

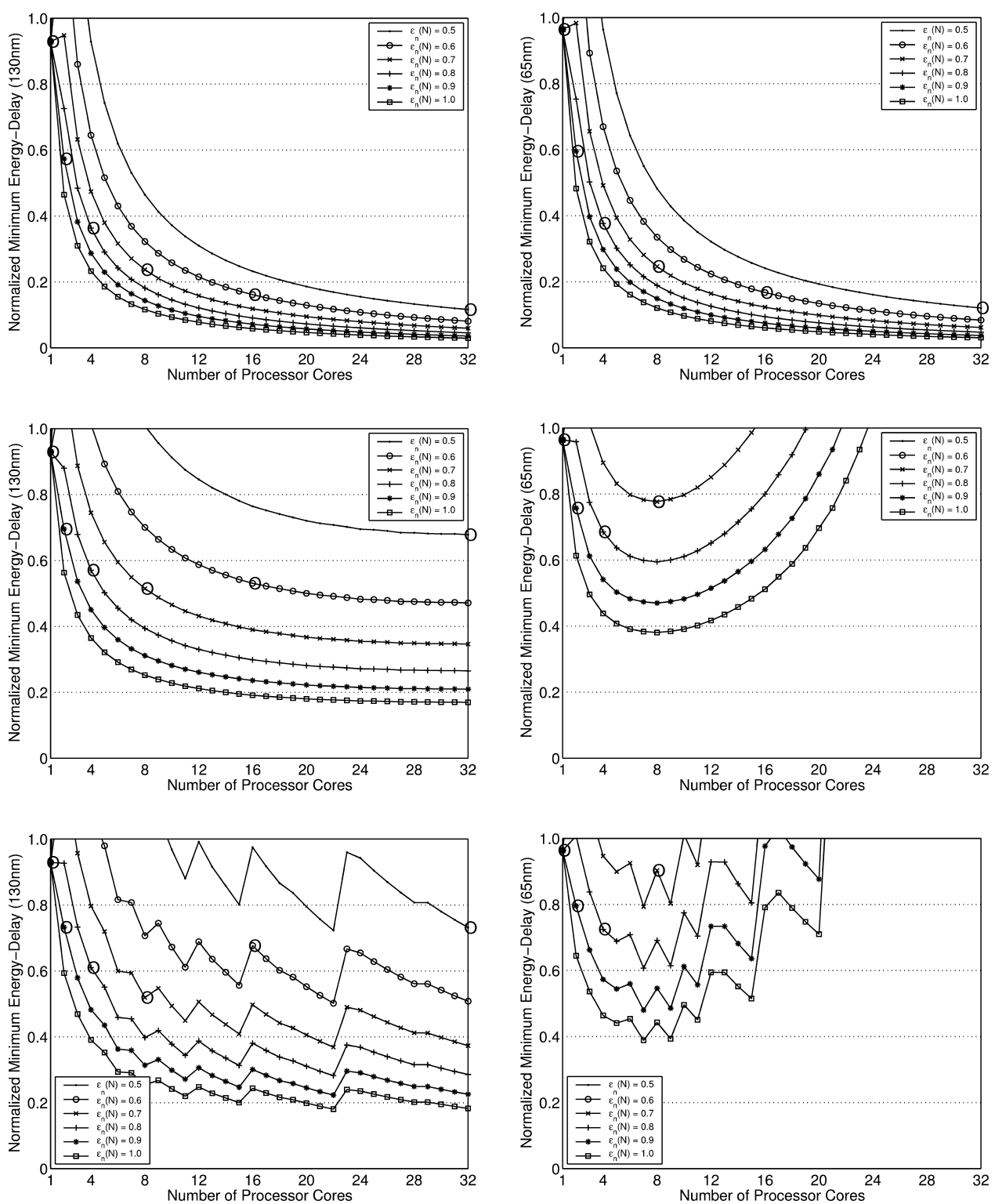

Fig. 3. Normalized minimum energy-delay product using voltage/frequency scaling with $130 \mathrm{~nm}$ (left) and $65 \mathrm{~nm}$ (right) technologies, $T_{1}=100^{\circ} \mathrm{C}$, and $N \in[1,32]$. Three cases are shown: Unbounded power budget and continuous voltage/frequency levels (top); bounded power budget (equal to the power consumption of the single-processor configuration at full throttle) and still continuous voltage/frequency levels (middle); and bounded power budget plus discrete voltage/frequency levels (up to 16 levels, equidistant) (bottom). Normalized minimum energy-delay product values greater than 1.0 are not shown. The circles in each plot indicate operating points for an imaginary application with $\varepsilon_{\mathrm{n}}(N)=\{1.0,0.9,0.8,0.7,0.6,0.5\}$. 
coincide with the full-throttle configuration). In other words, "local" (per-core) and "global" optima coincide when the parallel efficiency is 1 across all system sizes. In that case, $P_{N}=N P_{1}$, with $P_{1}$ the power consumption of the singleprocessor configuration that exhibits optimum energy-delay behavior. We can then write:

$$
E_{N} t_{N}=P_{N} t_{N}^{2}=N P_{1}\left(\frac{t_{1}}{N \varepsilon_{\mathrm{n}}(N)}\right)^{2}=\frac{1}{N} P_{1} t_{1}^{2}
$$

which indeed shows the $1 / N$ form.

In the other curves, for which $\varepsilon_{\mathrm{n}}(N)<1, N \in[2,32]$, the general shape is also along that of $1 / N$ for $N>1$, however, these curves are "lifted" by a factor that is proportional to the parallel efficiency in each case. This is most noticeable in the discontinuity going from $N=1$ to $N=2$ (most cases fall out of the plot), because of the fact that $\varepsilon_{\mathrm{n}}(1)$ is trivially 1 , and thus different from $\varepsilon_{\mathrm{n}}(N)$ for $N \in[2,32]$.

As in Scenario I (Section 2.3), however, the results acquire new meaning when an application with nonconstant parallel efficiency across $N$ is considered. Using the imaginary application from Scenario I with $\varepsilon_{\mathrm{n}}(N)=$ $\{0.9,0.8,0.7,0.6,0.5\}$ for $N=\{2,4,8,16,32\}$, respectively, we mark in the curves the resulting minimum for each $N$. The marks reveal a behavior not quite the same as the one displayed by the earlier curves, although the trend is still similar. This is about to change, however, as we introduce a power budget.

If the power budget is bounded (Figure 3, middle) -in our experiment, by the power consumption of the single-processor configuration at full throttle- the minimum energy-delay product curves exhibit a noticeable "lift" with respect to the earlier ones, for both 130 and $65 \mathrm{~nm}$. This is because of the limited applicable voltage/frequency scaling range introduced by the power budget limitation, which renders some of the earlier operating points unachievable. Furthermore, in $65 \mathrm{~nm}$, where static power becomes a significant fraction of the total power consumption, adding more processors can affect the minimum energy-delay product adversely, even if the parallel efficiency remains constant. In particular, the plot shows that an eight-processor configuration achieves the best energy-delay product among all the configurations $N \in[1,32]$.

As in the earlier case, when plotting the operating points of our imaginary application, the resulting trend is not quite the same as the curves with constant parallel efficiency. In fact, in the $130 \mathrm{~nm}$ case, the minimum energydelay product across all configurations is achieved at $N=8$, although the curves exhibit monotonic behavior. At $65 \mathrm{~nm}$, the optimum energy-delay product is obtained with a smaller $N=4$ configuration.

Finally, in the last experiment (Figure 3, bottom), we limit voltage/frequency scaling to sixteen discrete, equidistant levels, in addition to the already limited bounded power budget. As a result, the minimum energy-delay product curves lose continuity, exhibiting some degree of fluctuation. This has an adverse effect on the optimum energy-delay product of some of the application's operating points, sometimes quite noticeably, for both 130 and $65 \mathrm{~nm}$ technologies. 
Table I. CMP Configuration Modeled in the Experiments

\begin{tabular}{|l|l|}
\hline Process technology & $65 \mathrm{~nm}$ \\
Nominal frequency & $3.2 \mathrm{GHz}$ \\
Nominal $V_{\text {dd }}$ & $1.1 \mathrm{v}$ [The ITRS Technology Working Groups 2003] \\
$V_{\text {th }}$ & $0.18 \mathrm{v}$ [The ITRS Technology Working Groups 2003] \\
Ambient temperature & $45^{\circ} \mathrm{C}$ \\
Die Size & $244.5 \mathrm{~mm}^{2}(15.6 \mathrm{~mm} \times 15.6 \mathrm{~mm})$ \\
\hline CMP Size & 16 -way \\
Fetch/issue/commit width & $4 / 6 / 7$ \\
Inst. window [(Int+Mem)/FP] & $20 / 15$ \\
ROB & 80 \\
Ld/St queue & $32 / 32$ \\
Int/FP physical registers & $80 / 72$ \\
Int ALUs, mult/div & 4,2 \\
Branch units & 2 \\
FP ALUs, mult/div & 2,1 \\
Ld/St Units & 2 \\
Branch penalty (cycles) & 7 (min) \\
Store forward delay (cycles) & 2 \\
Branch predictor & Hybrid PAg/GAg with GAg chooser \\
BTB & $2 \mathrm{k}$-entry, 2-way \\
RAS & 32 -entry \\
\hline L1 I-, D-cache & $64 \mathrm{kB}, 64$ B line, 2-way, 2-cycle RT, \\
& 2 ports, WT/LRU \\
L1 MSHR entries & 16 \\
Unified L2 cache & Shared on chip, 4 MB, 128 B line, 8-way, \\
& 12 -cycle round-trip, 4 subbanks, 1 port, WB/LRU \\
I-TLB, D-TLB & Perfect \\
System bus & 256 bits, half processor speed \\
Max. outstanding requests & 64 \\
Memory bus bandwidth & $20 \mathrm{~GB} / \mathrm{s}$ \\
Memory & $75 \mathrm{~ns}$ round-trip (uncontended) \\
\hline
\end{tabular}

\section{EXPERIMENTAL SETUP}

To confirm the insights developed from the analytical model in Section 2 and assess its limitations, we conduct detailed simulations of parallel applications running on a detailed power-performance model of a CMP. In this section, we discuss the architecture modeled, the applications, and the power model.

\subsection{Architecture}

Our study uses a detailed model of a 16-processor CMP. These cores are modeled along the lines of the Alpha 21264 (EV6) processor [Compaq Computer Corporation 1999]. (While it is conceivable to consider a heterogeneous CMP model, its applicability and performance impact in the context of a parallel execution is beyond the scope of this paper.) Each processor core has private L1 instruction and data caches. All cores share a $4 \mathrm{MB}$ on-chip L2 cache through a common bus, and implement a MESI cache coherence protocol [Culler and Singh 1999]. Table I lists relevant cache and memory parameters.

We assume a 65-nm process technology. The original EV6 ran at $600 \mathrm{MHz}$ on a $350 \mathrm{~nm}$ process technology; by proceeding similarly to Kumar et al. [2003], we 
Table II. Applications Used in the Experiments

\begin{tabular}{|l|l|l|}
\hline Application & \multicolumn{1}{|c|}{ Description } & \multicolumn{1}{c|}{ Problem Size } \\
\hline Cholesky & Cholesky factorization kernel & tk15.O \\
\hline FFT & FFT kernel & 64 k points \\
\hline FMM & Adaptive fast multipole method & 16 k particles \\
\hline LU & LU kernel & $512 \times 512$ matrix, $16 \times 16$ blocks \\
\hline Ocean & Ocean movements & $514 \times 514$ ocean \\
\hline Radiosity & Iterative hierarchical diffuse radiosity method & room -ae 5000.0 -en 0.05 -bf 0.1 \\
\hline Radix & Integer radix sort kernel & $1 \mathrm{M}$ integers, radix 1024 \\
\hline Raytrace & 3-D ray tracing & car \\
\hline Volrend & Volume render using a ray casting technique & head \\
\hline Water-Ns & Forces and potentials of water molecules & 512 molecules \\
\hline BSOM & Batched self-organizing maps & 16 k records, 104 dimensions \\
& & 16 -node network, 50 iterations \\
\hline Equake & Earthquake modeling & train, 50 iterations \\
\hline Gafort & Genetic algorithm in Fortran & test \\
\hline SWIM & Shallow water modeling & train, 50 iterations \\
\hline
\end{tabular}

determine the clock frequency of our $65-\mathrm{nm}$ EV6-like cores to be $3.2 \mathrm{GHz}$. We set nominal supply and threshold voltages at 1.1 and $0.18 \mathrm{v}$, respectively [The ITRS Technology Working Groups 2003] and in-box ambient air temperature at $45^{\circ} \mathrm{C}$ [Majan 2002; Skadron et al. 2003]. Using CACTI [Wilton and Jouppi 1996], we obtain an estimated chip area of $244.5 \mathrm{~mm}^{2}(15.6 \times 15.6 \mathrm{~mm})$, using a scaling method similar to Kumar et al. [2004].

For the sake of simplicity, we assume global voltage/frequency scaling for the entire chip. (While it is conceivable to allow each core to run at a different frequency, the applicability and performance impact in the context of a parallel execution is nontrivial and beyond the scope of this paper.) Frequency can scale from $3.2 \mathrm{GHz}$ down to $200 \mathrm{MHz}$, and we resort to Intel Corporation [2004] to establish the relationship between frequency and supply voltage. Notice that, because voltage/frequency scaling is applied at the chip level, on-chip latencies (e.g., on-chip cache hit time) do not vary in terms of cycles. However, a round trip to off-chip memory takes the same amount of time regardless of the voltage/ frequency scaling applied on chip. Thus, the round-trip memory latency in processor cycles goes down as we downscale voltage and/or frequency. This is unlike the analytical model, where a system-level voltage/frequency scaling is assumed (Section 2).

\subsection{Applications}

We use fourteen parallel applications from various problem domains: Cholesky, FFT, FMM, LU, Ocean, Radiosity, Radix, Raytrace, Volrend, and Water-Ns from the SPLASH-2 application suite [Woo et al. 1995]; Equake, Gafort, and SWIM from the SPEC OMPM2001 suite [Aslot and Eigenmann 2003]; and BSOM, which is a parallel implementation of a data-mining application [Lawrence et al. 1999]. Table II lists the applications and their execution parameters. In the experiments, the problem sizes are generally smaller than real-life sizes, to allow for reasonable simulation times. Where a reduced input set was not directly available (e.g., BSOM), we carefully engineered one after 
studying the application. Problem sizes remain constant regardless of $N$ in all cases. For all applications, we skip initialization and then simulate to completion.

\subsection{Power Model}

We use Wattch to model the switching activity and dynamic power consumption of the on-chip functional blocks. This is different from our analytical model (Section 2), where we assumed a constant activity factor $A=1$ for all on-chip circuitry (Eq. 2). As for static power consumption, we model it as a fraction of the dynamic power consumption [Chaparro et al. 2004; Skadron et al. 2003]. In our model, this fraction is exponentially dependent on the temperature [Chaparro et al. 2004]. We use the HotSpot thermal model [Skadron et al. 2003] for chip temperature estimation. The average operating temperature, measured over a four-by-four array of 16 EV6 floorplan tiles downscaled to $65 \mathrm{~nm}$ [Skadron et al. 2003], ranges from in-box ambient air temperature $\left(45^{\circ} \mathrm{C}\right)$ to a maximum operating temperature of $100^{\circ} \mathrm{C}$, in agreement with multiple contemporary processor chip designs.

Wattch is reasonably accurate in relative terms; however, the absolute power values can be off by a nontrivial amount [Kumar et al. 2003]. Because we use power values to communicate across two different tools (Wattch and HotSpot), we ought to ensure that it is done in a meaningful way. We achieve this by renormalizing power values as follows.

We use HotSpot to determine the maximum operational power consumption (dynamic plus static), which is the one that yields the maximum operating temperature of $100^{\circ} \mathrm{C}$. Then, using the dynamic/static ratio that corresponds to that temperature [Chaparro et al. 2004], we derive the dynamic component.

We now need to establish the connection with Wattch. To do so, we use a compute-intensive microbenchmark to recreate a quasi-maximum power consumption scenario at nominal voltage and frequency levels in our simulation model, and obtain Wattch's dynamic power value. This number is often different from the one obtained through HotSpot using the method explained above. To overcome this gap, we calculate the ratio between Wattch and HotSpot's dynamic power values and use it throughout the experiments to renormalize wattage obtained with Wattch in our simulations as needed. This makes it possible for both tools to work together. While the absolute power may again not be exact, the results should be meaningful in relative terms. Using both tools, plus the power ratio/temperature curve, we are able to connect dynamic and static power consumption with temperature for any voltage and frequency scaling point.

Finally, we notice that the temperature and power density of the shared L2 cache is significantly lower than the rest of the chip across all the applications studied. The reasons for this include: much less switching activity, aggressive clock gating in the model [Brooks et al. 2000], and large L2 cache dissipation area. This observation is in agreement with published work by others [Chaparro et al. 2004; Donald and Martonosi 2004]. To obtain meaningful figures of power density and temperature, we exclude L2 from the calculations. 
However, we do include the power consumption of L2 in the results for total power consumption.

\section{EVALUATION}

In this section we evaluate two scenarios, which correspond to the first two scenarios discussed in the analytical model (Section 2), namely, power and performance optimization, respectively. We then present an example exploration of the power-performance optimization space, using one application from our experimental setup to illustrate some of the possible challenges and opportunities in the context of our study.

\subsection{Scenario I: Power Optimization}

This scenario is analogous to Scenario I of the analytical model (Section 2.3). The goal is to find the configuration that maximizes power savings while delivering the same performance as the sequential execution at nominal voltage and frequency levels.

The simulation experiment is conducted conceptually similarly to Section 2.3. We first simulate the execution of all the applications on a number of processor cores ranging from one to sixteen, at nominal frequency and voltage levels (without regard to any power budget). Notice that some applications only work for a number of cores power of two and in that case we only study configurations with one, two, four, eight, and sixteen cores. From this, we obtain: (1) the nominal parallel efficiency curve for each application and (2) the power consumption of each application on the single-core configuration. The nominal parallel efficiency is then used to calculate the target frequency of each configuration (Eq. 7). The target voltage supply is extrapolated from Intel Corporation [2004], in each case. We then recalculate the simulation parameters that are sensitive to voltage/frequency scaling and run simulations again to collect power and performance statistics for processor cores. Figure 4 shows the simulation results. As in Section 2, we assume unused processor cores are turned off.

The first plot shows the nominal parallel efficiency values that we obtain during the profiling phase. We observe wide changes in nominal parallel efficiency levels, both within (as we change $N$ ) and across applications. In general, within an application, nominal parallel efficiency goes down as the number of processor cores goes up.

The second plot shows the actual speedups attained by the applications on the different configurations studied. That applications experience speedups seems counterintuitive, since the fundamental premise of this scenario is that all configurations are tuned to yield equal performance (that of the single-core configuration in each case). This is most noticeable with Ocean and, to a lesser extent, Radix, Cholesky, and others. One reason why this is the case is that as the number of processors increases and voltage/frequency scaling is applied to the chip (but not to off-chip memory), the processor-memory speed gap narrows, which benefits memory-bound applications. This is not captured by the analytical model in Section 2.3, which assumes system-wide voltage/frequency scaling. 

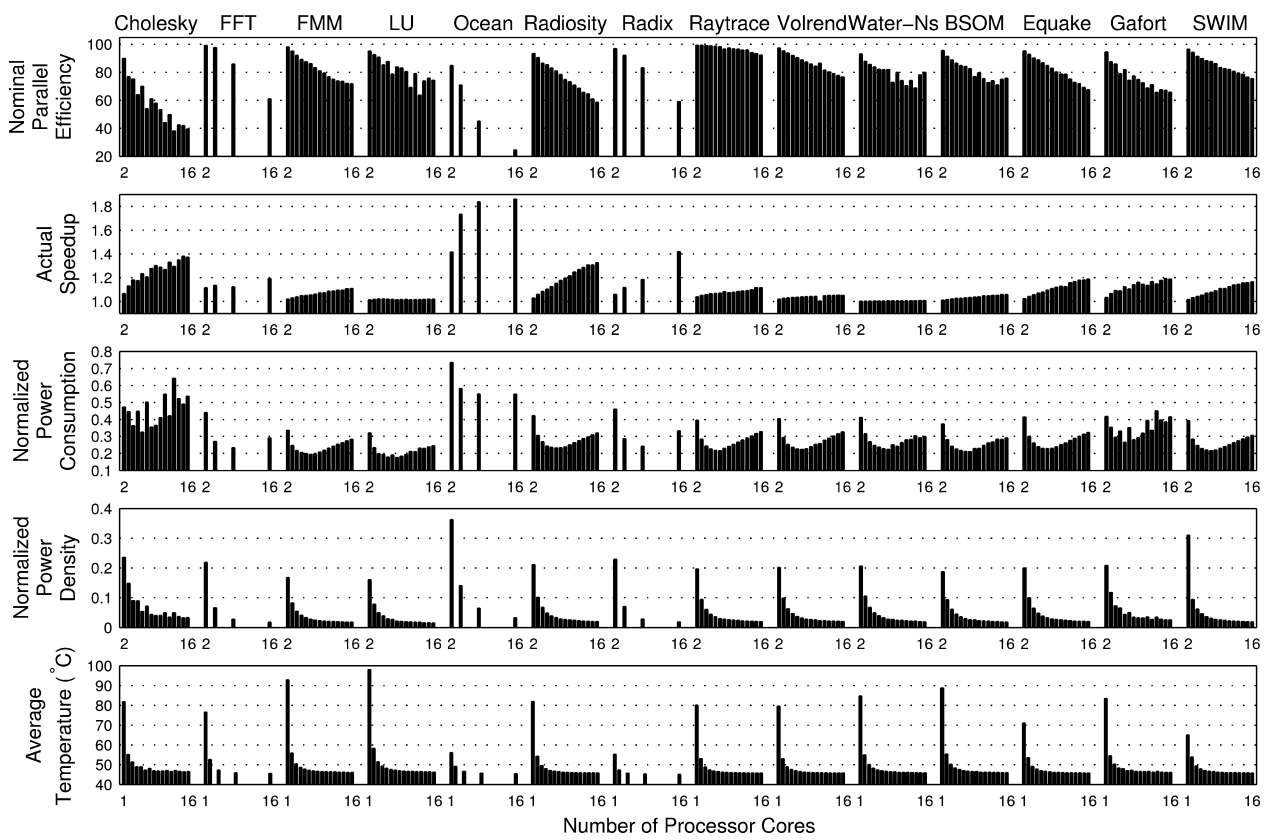

Fig. 4. Performance, power, and thermal characteristics of a 16-way CMP running the studied applications, according to the restrictions discussed in Scenario I (Section 4.1). Missing bars indicate that the corresponding application does not run with such a number of processors. All plots show results in the $N \in[2,16]$ range, except the temperature plot, which includes the $N=1$ case, as well.

The third plot shows, for each application, the power consumption in configurations of various $N$, normalized to the power consumption of the single-core configuration in each case. Given sufficient parallel efficiency, power consumption can be effectively reduced as the number of participating cores increases. Poor scalability, however, can make using more cores counterproductive. Indeed, the plot shows that, in general, a diminishing parallel efficiency resulting from increasing the number of processors eventually causes power savings to stagnate and, if even more cores are used, to actually recede. Our analytical model correctly predicts this behavior (Figure 1).

There are three main reasons behind this: First, the limited voltage range constrains the potential for power savings in relatively large configurations (Section 2). Second, as the parallel efficiency diminishes with increasing number of participating cores, the architecture cannot afford too aggressive voltage/ frequency levels if it is to meet the baseline performance. Third, the total static power consumption may increase as the number of processor cores increases (Eq. 9).

Another way to examine the effect of parallelization on power is to look at the power density. The fourth plot shows, for each application, the average power density for each $N$, normalized to the average power density for $N=1$ in each case. As we increase $N$, power density decreases rapidly as a result 
of aggressive voltage scaling (and consequent temperature drop). For example, in our experiments, we observe an across-the-board power density reduction of around $95 \%$ at $N=8$. As we approach the lower bounds of voltage and temperature, however, the reduction in power density becomes significantly slower.

Finally, the fifth plot shows the average operating temperature of the applications running on different configurations, including the single-core configurations. (Notice that, unlike the other four plots, this one is not normalized.) As a result of lower power density, the average operating temperature decreases as $N$ increases. Larger temperature reduction is observed in applications that consume more power at nominal operating voltage and frequency levels (FMM and LU). This is because of the exponential relation between temperature and static power. Again, the temperature reduction rate, initially very sharp, quickly slows down as $N$ increases.

Overall, we see that parallel computing can be an effective way to reduce chip power consumption, power density, and operating temperature on CMP given a performance target, although its effectiveness is very dependent on factors such as parallel efficiency, range of voltage/frequency scaling, and total leakage power, all of which tend to worsen as the number of participating cores goes up.

\subsection{Scenario II: Performance Optimization}

This scenario is analogous to the one in Section 2.4. In this case, we want to see the maximum speedup a $N$-processor configuration can achieve within the power budget of a single core, which we derive using microbenchmarking (Section 3.3). The simulation experiment is set up with off-line profiling, similarly to Section 4.1. In addition to obtaining profile information at the nominal frequency $(3.2 \mathrm{GHz})$, we obtain the power and performance statistics with frequencies ranging from $200 \mathrm{MHz}$ to $3.0 \mathrm{GHz}$, with a step of $200 \mathrm{MHz}$. We then use the profile information to calculate the optimal voltage and frequency in order to achieve the maximum speedup under the fixed power dissipation budget for each $N$-processor configuration. The configuration values that fall between any two profiled values are approximated by linearly scaling between the two. Using this information, we then run simulations again to get the real speedup.

We select three applications in this case study: FMM, Cholesky, and Radix, in descending order of computational intensity and power consumption. They are representative of the behavior observed in the all the applications considered. Both the nominal and the actual speedups of the three applications are presented in Figure 5. (The nominal speedup is derived from the nominal parallel efficiency values calculated in Section 4.1.)

As predicted by the analytical model (Section 2.4), we see a performance gap between the nominal configuration without considering power dissipation budget (nominal speedup) and the configuration that abides by the power budget constraint (actual speedup). The gap is most significant in the computeintensive application (FMM) and least so for Radix, which is memory-bound. In general, two situations help the performance scaling of memory-bound applications: First, as we apply voltage/frequency scaling to the chip-but not to off-chip memory-the processor-memory speed gap narrows, resulting in less 


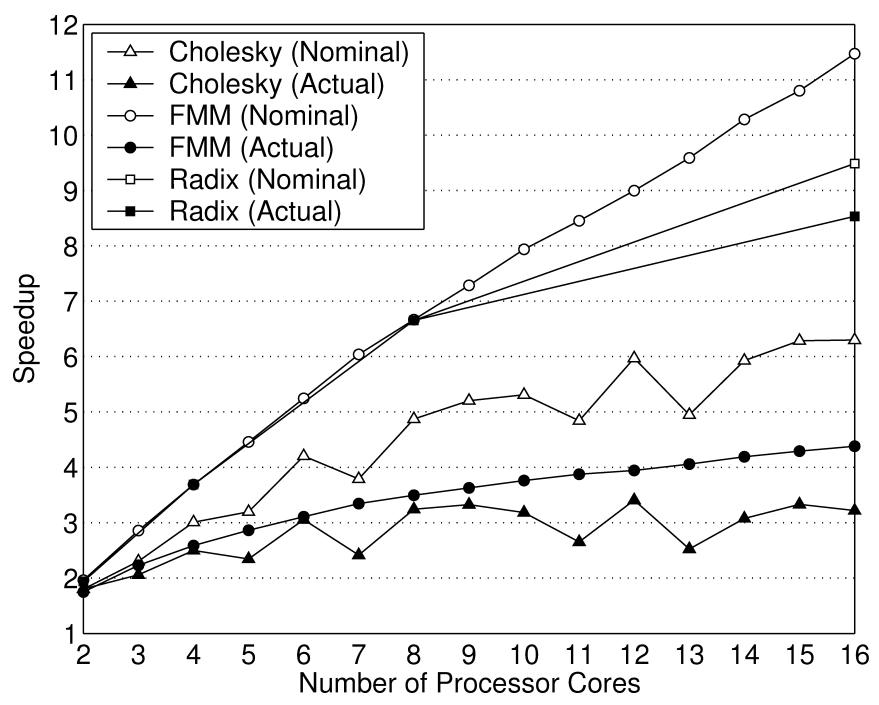

Fig. 5. Comparison of the nominal and actual speedup of three applications. The power budget is the maximum nominal power consumption of a single core (Section 3.3).

memory stalls. Second, due to stalling on memory accesses, these applications usually do not reach the chip's power budget when running sequentially. Thus, as we increase $N$, these applications can benefit from higher voltage/frequency levels and still abide by the power budget. As discussed previously, none of these effects is captured by the analytical model (Section 2). In fact, for up to eight processor cores, the actual speedup of Radix does match the nominal speedup (Figure 5). When looking at our data for these configurations, we find that they operate at nominal voltage and frequency levels. Indeed, the nominal power consumption of Radix is low enough that it allows up to eight-core configurations to run at nominal voltage and frequency without exceeding our power budget. However, as we continue increasing $N$, a gap between actual and nominal speedup eventually appears, as the configurations can no longer meet power budget constraints at nominal voltage and frequency levels.

Finally, notice that, for these memory-bound applications and low $N$, one could seek higher performance by overclocking the chip and still abide by the power budget. However, unless the memory subsystem is also overclocked, the resulting increase in the processor-memory speed gap could partially offset the potential performance gain.

\subsection{Power-Performance Space Exploration}

In this section, we conduct a short power-performance space exploration around BSOM, the parallel data mining application (Section 3.2), to illustrate some of the possible challenges and opportunities in the context of our study. Figure 6 shows the power consumption and execution time of an iteration of BSOM's main (parallel) loop, or epoch. Each point in the plot represents one CMP configuration, using a certain number of processors $N$ (shown in the legend) and a particular voltage/frequency level (not indicated directly in the plot). We vary $N$ 


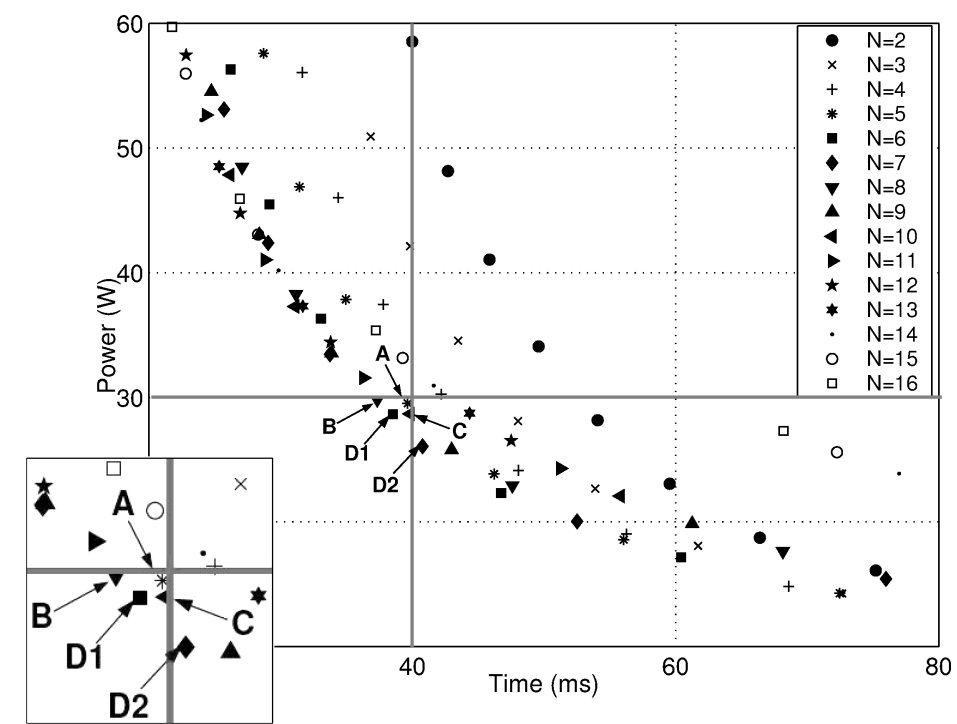

Fig. 6. Power consumption and execution time of CMP configurations with varying number of processors $N$ and voltage/frequency levels for an instance of BSOM's parallel region (a parallel data mining application). Target execution time and power are $40 \mathrm{~ms}$ and $30 \mathrm{~W}$, respectively.

between two and sixteen processors and, for each $N$, we explore sixteen different voltage/frequency levels, distributed regularly along the allowable range, $V_{1}$ to $2.3 V_{\text {th }}$. In the figure, we limit our discussion to a window of power ranging from 10 to $60 \mathrm{~W}$, and execution time of an epoch ranging from 20 to $80 \mathrm{~ms}$. (Naturally, we are using a reduced input set to make simulation time affordable; execution time of each epoch is longer with realistic input sizes.)

In general, as expected, points for a fixed $N$ move right and down as we reduce the voltage/frequency levels, as it takes more time to execute one epoch, but, on the other hand, the power consumption is lower. The plot shows two well-differentiated operating areas: On the one hand, operating points toward the upper-left corner lie in a performance-oriented (or power-sensitive) area. In this area, power consumption is generally high, but small reductions in voltage/ frequency levels translate into significant savings in power consumption, at a relatively small performance cost. On the other hand, operating points toward the lower-right corner lie in a power-oriented or (performance-sensitive) area. In this area, while performance is generally low, small voltage/frequency increases result in large performance gains, at a relatively small power cost.

In a situation typical of Scenario I (Section 2.3), for example, where we impose a performance target and seek to minimize power consumption, we can again find two well-differentiated areas. As we move left (strict performance target), configurations with a small number of processors need to operate at high voltage/frequency levels to meet the performance target, thus consuming significant power. If, as it is the case in BSOM, parallel efficiency is generally high, a higher number of processors allows the CMP to meet the performance target while achieving significant power savings by aggressively throttling voltage and frequency. On the other hand, toward the right end of the plot (relaxed 
performance target), most configurations can meet or exceed the performance target with very low voltage and frequency levels. In that case, the static power consumption of configurations with a high number of processors puts them at a disadvantage with respect to smaller configurations.

If we assume the target execution time for one epoch is $40 \mathrm{~ms}$ (vertical line) and we further consider $30 \mathrm{~W}$ as the maximum allowable power dissipation (horizontal line), our search for valid configurations is confined, in principle, to the quadrant left of and below such bounds, respectively. Only a few operating points reside in this quadrant, which we label A, B, C, and D1 in the plot. Any of these points satisfy both performance and power constraints; the actual choice depends on a number of possible factors.

Configuration $\mathrm{C}$ in the plot, for example, yields acceptable performance and maximum power savings. Configuration D1 yields better performance at the same power cost and thus, would seem a better choice in terms of overall powerperformance behavior. Configurations $\mathrm{A}$ and $\mathrm{B}$ consume a little more power, but are still within the allowed limit.

One difference between these operating points lies in the number of processors required by each case, which varies widely-between five (configuration A) and ten (configuration $\mathrm{C}$ ) in our experiment. If the application is also expected to minimize the number of allocated processors (for example, in a multiprogrammed environment), configuration $\mathrm{C}$ may prove too costly at $N=10$. On the other hand, configuration A, which uses one-half as many processors, may constitute an attractive choice in this case, even as it consumes slightly more power. The opposite may be true if, for example, power density is a concern.

Finally, if we define our performance target as a rate (e.g., frames per second in MPEG decoder/encoder) and allow epochs to borrow leftover time from previous "fast" ones to execute slower and save more power while still meeting the target rate, further power savings opportunities may be possible. For example, we can alternate runs with $N=6$ for one epoch and $N=7$ for the following epoch (configurations D1 and D2 in the plot, respectively). These configurations result, as the plot shows, in per-epoch execution times that are slightly under and over target, respectively. However, alternating between these two states is likely to meet or exceed the target rate (with D2 borrowing time from the slack created by D1). The combination D1-D2 consumes, on average, less power than any other valid configuration by itself. Therefore, it can be argued that configuration D1 controls execution time as configuration D2 lowers power consumption, resulting in a favorable net balance. Notice, however, that this setup may require more sophisticated analysis and support. Among other issues, the application must be able to change the number of processors dynamically across epochs.

Overall, this example shows that there is great potential in trying to exploit knowledge about the power-performance optimization space of an application running on a particular CMP. The challenge resides precisely in constructing such knowledge (or a useful subset), for example, dynamically at run time in a manner that does not significantly disrupt the application's execution or affect its performance adversely (e.g., by intelligently steering the exploration of the possible operating points) and in a way largely transparent to the programmer 
or compiler (e.g., by leveraging existing parallel constructs for which the number of processors involved is not explicitly stated by the programmer or compiler, which would facilitate exploratory/adaptive behavior). The particular mechanisms to construct and exploit such knowledge represent future work.

\section{RELATED WORK}

Earlier VLSI works have discussed the trade-offs that sequential vs. parallel circuits present in silicon area and power consumption [Chandrakasan et al. 1992; Parhi 1999]. However, thus far, very little work has been done on the power-performance issues involving parallel applications executing on multiprocessors, in general and on multicore chips, in particular.

There is rich literature on power/thermal-aware simultaneous multithreading (SMT) and CMP designs (or similar architecture configurations), most of which focuses on multiprogrammed workloads [Donald and Martonosi 2004; Ghiasi and Grunwald 2004; Kumar et al. 2003; Li et al. 2004, 2005; Sasanka et al. 2004; Seng et al. 2000]. In contrast, our work focuses on the powerperformance issues of CMPs in the context of parallel applications. In particular, a fundamental contribution of our work is the formal connection between parallel efficiency of the application and the power-performance behavior at each operating point, whereas the concept of parallel efficiency is meaningless (and thus cannot be captured) in a multiprogrammed context.

Huh et al. [2001] conduct an in-depth exploration of the design space of CMPs. However, they do not address power. More recently, Ekman and Stenström [2003] conduct a design-space study of CMPs in which they address some power issues. Assuming a certain silicon budget, they compare chips with different numbers of cores and correspondingly different core sizes. They argue that parallel applications with limited scalability, but some instruction-level parallelism may run better on CMPs with few, wide-issue cores. They also argue that CMPs with few, wide-issue cores and with many, narrow-issue cores consume roughly the same power, as cache activity offsets savings at the cores. Our work assumes a given chip design and explores the issues of assigning different numbers of cores to a parallel application, given certain power-performance constraints. Their work also uses $0.18 \mu \mathrm{m}$ process technology and does not consider voltage/frequency scaling, which is a fundamental component in our work.

Grochowski et al. [2004] discuss trade-offs between microprocessor processing speed vs. throughput in a power-constrained environment. They postulate that a microprocessor that can achieve both high scalar performance and high throughput performance ought to be able to dynamically vary the amount of energy expended to process each instruction, according to the amount of parallelism available in the software. To achieve this, they survey four techniques: dynamic voltage/frequency scaling (DVFS), asymmetric cores, variable-sized cores, and speculation control, and conclude that a combination of DVFS and asymmetric cores is best.

More recently, Annavaram et al. [2005] use asymmetric CMP to maximize the performance of a multithreaded application by assuming nontrivial sequential components exist in the application. They use the notion of EPI (energy per instruction) throttle to orchestrate the application's execution on its sequential 
and parallel portions under a fixed power budget. For the sequential portions, they assign a faster but more power-hungry processor. For the parallel portions, depending on the number of threads that are inherent in the application's parallelization and the number of available processors, they assign multiple slower, but power-thrifty, processors. In our work, we formally connect granularity of parallelism, application's parallel efficiency, and DVFS to work three scenarios on a symmetric CMP: performance optimization under a power budget constraint, power optimization given a performance target, and energy-delay product optimization.

Kaxiras et al. [2001] compare the power consumption of an SMT and a CMP digital signal-processing chip for mobile phone applications. They do not explicitly study parallel applications in the "traditional" sense. For example, they approximate a parallel encoder with four independent MPEG encoder threads, each thread processing one quarter of the original image size. A speech encoder and decoder are connected in a pipelined fashion to a channel encoder and decoder, respectively. The issues of granularity vs. parallel efficiency and DVFS that we address cannot be easily conveyed in this context.

Kadayif et al. [2002] propose to shut down idle processors in order to save energy when running nested loops on a CMP. The authors also study a preactivation strategy based on compiler analysis to reduce the wake-up overhead of powered-off processors. Although they address program granularity and power, they do not exploit DVFS in their solution, which is fundamental in our work.

In a different work, Kadayif et al. [2004] propose to use DVFS to slow down lightly loaded threads to compensate for load imbalance in a program and save power and energy. They use the compiler to estimate the load imbalance of array-based loops on single-issue processor cores. The authors also mention the opportunity of further energy savings by using less than the number of available processor cores using profile information. However, the connection of DVFS to granularity and parallel efficiency of the code is not fleshed out.

In the context of cache-coherent shared-memory multiprocessors, Moshovos et al. [2001] reduce energy consumption by filtering snoop requests in a bus-based parallel system. Saldanha and Lipasti [2001] observe significant potential of energy savings by using serial snooping for load misses. Li et al. [2004] propose to save energy wasted in barrier spin-waiting, by predicting a processor's stall time and, if warranted, forcing it into an appropriate ACPI-like lowpower sleep state. This last work is complementary to ours in that it targets energy reduction during wasteful processor activity, whereas our work attacks power consumption during useful activity by the application.

In an environment of loosely coupled web servers running independent workloads, several studies evaluate different policies to control the number of active servers (and thus their performance level) to preserve power while maintaining acceptable quality of service [Elnozahy et al. 2002, 2003; Pinheiro et al. 2001; Rajamani and Lefurgy 2003].

In the context of micro-architectures, Heo and Asanović [2004] study the effectiveness of pipelining as a power-saving tool in a uniprocessor. They examine the relationship between the logic depth per stage and the supply voltage in deep submicron technology under different conditions. This is complementary 
to our work, since we study power-performance issues of using multiple cores on a CMP.

\section{CONCLUDING REMARKS}

In this paper, we have explored power-performance issues of running parallel applications on a CMP. We have developed an analytical model to study the effect of combining granularity, parallel efficiency, and voltage/frequency scaling on the performance and power consumption delivered by a CMP. To confirm the insights developed from the analytical model and assess its limitations, we have conducted detailed simulations of parallel applications running on a power-performance model of a CMP. The experiments confirm that the analytical model captures the power-performance behavior reasonably well.

Both analytical and experimental models show that, through judicious choice of granularity and voltage/frequency scaling, parallel computing can bring significant power savings while meeting a given performance target. The particular choice, however, is dependent on the application's parallel efficiency curve and the process technology utilized, which our model captures.

Similarly, analytical model and experiments show the effect of a limited power budget on the application's scalability curve. In particular, we have shown that a limited power budget can cause a rapid performance degradation beyond a number of cores, even in the case of applications with excellent scalability properties. On the other hand, our experiments have shown that power-thrifty memory-bound applications can actually enjoy better scalability than more nominally scalable applications when a limited power budget is in place.

Overall, our study shows that, under the right circumstances, parallel computing may bring significant power-performance benefits over a uniprocessor setup of similar performance. However, it also illustrates the dependency of the optimum operating point on multiple interacting factors, such as the application's parallel efficiency, the chip's voltage/frequency scaling characteristics, the process technology, and restrictions in performance, power, and, sometimes, number of available processors. Our study shows that these factors can interact in a nonobvious way, making power-performance optimization of on-chip parallel computation quite challenging. In all, we believe that the insights developed in this paper form a compelling case for research efforts on hardware-software mechanisms that address this problem.

\section{ACKNOWLEDGMENTS}

We thank David Albonesi, Rajit Manohar, and the anonymous reviewers for useful feedback. This work was supported in part by NSF awards CNS-0509404, CCF-0429922, and gifts from Intel. Jian Li was supported in part by NSF awards CNS-0406345 and ACI-0121401.

\section{REFERENCES}

Agerwala, T. And Chatterjee, S. 2005. Computer architecture: Challenges and opportunities for the next decade. IEEE Micro 25, 3 (May-June), 58-69.

ACM Transactions on Architecture and Code Optimization, Vol. 2, No. 4, December 2005. 
Annavaram, M., Grochowski, E., And Shen, J. 2005. Mitigating Amdahl's Law through EPI throttling. In International Symposium on Computer Architecture, Madison, Wisconsin, 298-309.

Aslot, V. And Eigenmann, R. 2003. Quantitative performance analysis of the SPEC OMPM2001 benchmarks. Scientific Programming 11, 2, 105-124.

BorkaR, S. 1999. Design challenges for technology scaling. IEEE Micro 19, 4 (July-Aug.), 23-29.

Brooks, D., Tiwari, V., AND Martonosi, M. 2000. Wattch: A framework for architectural-level power analysis and optimizations. In International Symposium on Computer Architecture, Vancouver. 83-94.

Chandrakasan, A., Sheng, S., and Brodersen, R. W. 1992. Low-power CMOS digital design. IEEE Journal of Solid-State Circuits 27, 4 (Apr.), 473-484.

Chaparro, P., GonzÁlez, J., ANd GonzÁlez, A. 2004. Thermal-effective clustered microarchitectures. In Workshop on Temperature-Aware Computer Systems, München.

Compaq Computer Corporation. 1999. Alpha 21264 Microprocessor Hardware Reference Manual. Compaq Computer Corp., Shrewsbury, MA.

Culler, D. E. And Singh, J. P. 1999. Parallel Computer Architecture: A Hardware/Software Approach. Morgan Kaufmann.

Donald, J. ANd Martonosi, M. 2004. Temperature-aware design issues for SMT and CMP architectures. In Workshop on Complexity-Effective Design, München.

Ekman, M. And Stenström, P. 2003. Performance and power impact of issue-width in chipmultiprocessor cores. In International Conference on Parallel Processing, Kaohsiung. 359-368.

Elnozahy, E. N., Kistler, M., ANd RAJAmony, R. 2002. Energy-efficient server clusters. In Workshop on Power Aware Computing Systems. Cambridge, MA. 179-196.

Elnozahy, E. N., Kistler, M., and Rajamony, R. 2003. Energy conservation policies for web servers. In USENIX Symposium on Internet Technologies and Systems. Seattle, WA.

Ghiasi, S. ANd Grunwald, D. 2004. Design choices for thermal control in dual-core processors. In Workshop on Complexity-Effective Design, München.

Gonzalez, R. AND HoRowitz, M. 1996. Energy dissipation in general purpose microprocessors. IEEE Journal of Solid-State Circuits 31, 9 (Sept.), 1277-1284.

Grochowski, E., Ronen, R., Shen, J., And Wang, H. 2004. Best of both latency and throughput. In International Conference on Computer Design, San Jose, CA. 236-243.

Gunther, S., Binns, F., Carmean, D. M., and Hall, J. C. 2001. Managing the impact of increasing microprocessor power consumption. Intel Technology Journal 5, 1 (Feb.).

Hennessy, J. L. and Patterson, D. A. 2003. Computer Architecture: A Quantitative Approach, 3rd edn. Elsevier Science, New York.

Heo, S. and Asanović, K. 2004. Power-optimal pipelining in deep submicron technology. In International Symposium on Low Power Electronics and Design, Newport Beach, CA.

Huh, J., Burger, D., AND Keckler, S. W. 2001. Exploring the design space of future CMPs. In International Conference on Parallel Architectures and Compilation Techniques, Barcelona. 199210.

Intel Corporation. 2004. Intel Pentium M Processor on 90nm Process with 2-MB L2 Cache Datasheet. Intel Corp. Hudson, MA.

KADAYIF, I., KANDEMIR, M., AND SEZER, U. 2002. An integer linear programming based approach for parallelizing applications in on-chip multiprocessors. In IEEE/ACM Design Automation Conference, New Orleans, LA. 703-708.

KADAYif, I., KANDEmiR, M., ViJaykRishnan, N., AND IRwin, M. J. 2004. Exploiting processor workload heterogeneity for reducing energy consumption in chip multiprocessors. In Design, Automation and Test in Europe, Paris. 1158-1163.

Kaxiras, S., Narlikar, G., Berenbaum, A. D., and Hu, Z. 2001. Comparing power consumption of an SMT and a CMP DSP for mobile phone workloads. In International Conference on Compilers, Architecture, and Synthesis for Embedded Systems, Atlanta, Georgia. 211-220.

Kim, N. S., Austin, T., Blaauw, D., Mudge, T., Flautner, K., Hu, J. S., Irwin, M. J., Kandemir, M., and Narayanan, V. 2003. Leakage current: Moore's law meets static power. IEEE Computer 36, 12 (Dec.), 68-75.

Kumar, R., Farkas, K. I., Jouppi, N. P., Ranganathan, P., and Tullsen, D. M. 2003. Single-ISA heterogeneous multi-core architectures: The potential for processor power reduction. In International Symposium on Microarchitecture, San Diego, CA. 81-92. 
Kumar, R., Tullsen, D. M., Ranganathan, P., Jouppi, N. P., and Farkas, K. I. 2004 . Single-ISA heterogeneous multi-core architectures for multithreaded workload performance. In International Symposium on Computer Architecture, München. 64-75.

Lawrence, R. D., Almasi, G. S., AND Rushmeier, H. E. 1999. A scalable parallel algorithm for selforganizing maps with applications to sparse data mining problems. Data Mining and Knowledge Discovery 3, 2 (Sept.), 171-195.

Li, J., MartíneZ, J. F., AND HuAng, M. C. 2004. The Thrifty Barrier: Energy-aware synchronization in shared-memory multiprocessors. In International Symposium on High-Performance Computer Architecture, Madrid. 14-23.

Li, Y., Skadron, K., Brooks, D., AND Hu, Z. 2004. Understanding the energy efficiency of simultaneous multithreading. In International Symposium on Low Power Electronics and Design, Newport Beach, CA. 207-212.

Li, Y., Brooks, D., Hu, Z., ANd Skadron, K. 2005. Performance, energy, and temperature considerations for SMT and CMP architectures. In International Symposium on High-Performance Computer Architecture, San Francisco, CA.

MAJAN, R. 2002. Thermal management of CPUs: A perspective on trends, needs and opportunities. In International Workshop on Thermal Investigations of ICs and Systems, Madrid. (Keynote presentation).

Moshovos, A., Memik, G., Falsafi, B., and Choudhary, A. 2001. JETTY: Filtering snoops for reduced energy consumption in SMP servers. In International Symposium on High-Performance Computer Architecture, Nuevo Leone. 85-96.

Mudge, T. 2001. Power: A first-class architectural design constraint. IEEE Computer 34, 4 (Apr.), $52-58$.

PARHI, K. K. 1999. VLSI Digital Signal Processing Systems. Wiley, New York.

Pinheiro, E., Bianchini, R., Carrera, E., And Heath, T. 2001. Load balancing and unbalancing for power and performance in cluster-based systems. In International Workshop on Compilers and Operating Systems for Low Power, Barcelona.

RAJAMANI, K. AND LEFURGY, C. 2003. On evaluating request-distribution schemes for saving energy in server clusters. In International Symposium on Performance Analysis of Systems and Software, Austin, TX. 111-122.

Saldanha, C. and Lipasti, M. 2001. Power efficient cache coherence. In Workshop on Memory Performance Issues, Göteborg.

Sasanka, R., Adve, S. V., Chen, Y., and Debes, E. 2004. Comparing the energy efficiency of CMP and SMT architectures for multimedia workloads. In International Conference on Supercomputing, Malo. 196-206.

Seng, J. S., Tullsen, D. M., And Cai, G. Z. N. 2000. Power-sensitive multithreaded architecture. In International Conference on Computer Design, Austin, Texas. 199-208.

Skadron, K., Stan, M., Huang, W., and Velusamy, S. 2003. Temperature-aware microarchitecture: Extended discussion and results. Tech. Rep. CS-2003-08, University of Virginia. (Apr.)

The ITRS Technology Working Groups. 2003. International Technology Roadmap for Semiconductors (ITRS). The ITRS Technology Working Groups.

Weiser, U. 2004. Microprocessors: Bypass the power wall. In Intel Academic Forum, Barcelona. (Keynote presentation).

Wilton, S. AND JoupPI, N. 1996. CACTI: An enhanced cache access and cycle time model. IEEE Journal of Solid-State Circuits 31, 5 (May), 677-688.

Woo, S. C., Ohara, M., Torrie, E., Singh, J. P., And Gupta, A. 1995. The SPLASH-2 programs: Characterization and methodological considerations. In International Symposium on Computer Architecture. Santa Margherita Ligure, Italy. 24-36.

Zyuban, V., Brooks, D., Srinivasan, V., Gschwind, M., Bose, P., Strenski, P. N., and Emma, P. G. 2004. Integrated analysis of power and performance for pipelined microprocessors. IEEE Transactions on Computers 53, 8 (Aug.), 1004-1016.

Received August 2005; revised November 2005; accepted December 2005 